\title{
The star-formation rate in the host of GRB 990712
}

\author{
P. M. Vreeswijk ${ }^{1}$, R. P. Fender ${ }^{1}$, M. A. Garrett ${ }^{2}$, S. J. Tingay ${ }^{3}$, A. S. Fruchter ${ }^{4}$, and L. Kaper ${ }^{1}$ \\ 1 Astronomical Institute "Anton Pannekoek", University of Amsterdam \& Center for High Energy Astrophysics, \\ Kruislaan 403, 1098 SJ Amsterdam, The Netherlands \\ 2 Joint Institute for VLBI in Europe (JIVE), Postbus 2, 7990 AA, Dwingeloo, The Netherlands \\ 3 Australia Telescope National Facility, Paul Wild Observatory, Locked Bag 194, Narrabri, NSW 2390, Australia \\ 4 Space Telescope Science Institute, 3700 San Martin Drive, Baltimore, MD 21218, USA
}

Received 13 August 2001 / Accepted 23 October 2001

\begin{abstract}
We have observed the host galaxy of GRB 990712 at $1.4 \mathrm{GHz}$ with the Australia Telescope Compact Array, to obtain an estimate of its total star-formation rate. We do not detect a source at the position of the host. The $2 \sigma$ upper limit of $70 \mu \mathrm{Jy}$ implies that the total star-formation rate is lower than $100 M_{\odot} \mathrm{yr}^{-1}$, using conservative values for the spectral index and cosmological parameters. This upper limit is in stark contrast with recent reports of radio/submillimeter-determined star-formation rates of $\sim 500 M_{\odot} \mathrm{yr}^{-1}$ for two other GRB host galaxies. Our observations present the deepest radio-determined star-formation rate limit on a GRB host galaxy yet, and show that also from the unobscured radio point-of-view, not every GRB host galaxy is a vigorous starburst.
\end{abstract}

Key words. gamma rays: bursts - radio continuum: galaxies - stars: formation

\section{Introduction}

The gamma-ray bursts (GRBs) for which afterglows have been observed so far, i.e. bursts with a duration longer than roughly two seconds, can be most adequately explained by the collapse of a rapidly rotating, massive star. In this collapsar model (Woosley 1993; Paczyński 1998; MacFadyen \& Woosley 1999; MacFadyen et al. 2001), the GRB is produced in narrow cones along the rotational axis of the collapsing progenitor, accompanied by an isotropic supernova explosion of a type similar to SN 1998bw (Galama et al. 1998). For a few GRBs the presence of a supernova has been inferred from a bump in the optical afterglow light curve at late times (Bloom et al. 1999a; Reichart 1999; Galama et al. 2000). Moreover, the locations of GRB afterglows coincide with the optical extent of their host galaxies (Bloom et al.2001c; Fruchter et al., in prep.), suggesting that these long-duration GRBs are linked with regions of star formation. The observed GRB location distribution is not expected for the alternative binary neutron star merger model (or a neutron star and a black hole) (Eichler et al. 1989; Narayan et al. 1992), where the kick velocities received from the two supernovae and the time it takes the two compact objects to merge, would cause the GRB to occur kiloparsecs away from the place of birth of the progenitor binary

Send offprint requests to: P. M. Vreeswijk

e-mail: pmv@astro.uva.nl
(Bloom et al. 1999b), in at least a few cases. These mergers, however, are expected to be the progenitors of the category of short-duration GRBs (Fryer et al. 1999).

In case the gamma rays come from internal shocks, which is the generally favoured model, a GRB can be observed both in the case of a collapsar and of a merger, i.e. irrespective of its environment. Afterglows are thought to be produced by the interaction of the fireball ejecta with the environment (the flux in the fireball model is proportional to the square root of the density of the circumburst medium, e.g. Wijers \& Galama 1999). If mergers would also produce long-duration GRBs, we would have expected a fraction of these to have no X-ray afterglow - namely those which occur outside a galaxy. However, nearly all attempts to detect an X-ray afterglow were successful (e.g. Stratta et al. 2000), which suggests that they are not the result of mergers (assuming the internal shock model). Note that the location argument in favour of the collapsar model does not necessarily hold if the gamma rays are produced by external shocks. In that case the gamma rays themselves are produced by interaction with the circumburst medium, which may mean that all GRBs (both long and short-durations bursts) and their afterglows that occur in low-density environments are not detected.

If GRBs are intimately connected with the deaths of massive stars, they are potential probes of star formation in the early universe. At present, it is not known which type of galaxy produces the bulk of star formation at high 
redshift: the numerous faint blue galaxies (Ellis 1997), or the ultra-luminous infrared or starburst galaxies (Sanders \& Mirabel 1996). Determination of the type of galaxy that gives birth to GRBs can provide important clues to this outstanding issue.

Star-formation rates $(S F R \mathrm{~s})$ for several GRB host galaxies have been estimated from optical nebular emission lines (e.g. [OII]) to vary from $0.3 M_{\odot} \mathrm{yr}^{-1}$ for the host of GRB 970828 (Djorgovski et al. 2001) to $24 M_{\odot} \mathrm{yr}^{-1}$ for the host of GRB 980703 (Djorgovski et al. 1998). These values are not yet corrected for dust extinction, which is difficult to estimate and which can be quite large. This causes considerable uncertainty in the $S F R$ values. Recently, very high star-formation rates $\left(\sim 500 M_{\odot} \mathrm{yr}^{-1}\right)$, have been inferred for GRB 980703 and GRB 010222, using radio (Berger et al. 2001) and submillimeter (Frail et al. 2001) measurements, respectively, which do not suffer from dust extinction. The question that arises is: do all GRB host galaxies look like vigorous starburst galaxies when they are observed at the unobscured radio and submillimeter wavelengths?

Due to its relative proximity $(z=0.433)$, the host of GRB 990712 is an excellent GRB host galaxy to study in detail. VLT spectra of the host show that the galaxy is an HiI galaxy (i.e. the spectral emission lines are produced by HII regions that are being ionized by $\mathrm{O}$ and $\mathrm{B}$ stars) and not a galaxy that is hosting an active galactic nucleus (AGN). The [OII] emission star-formation rate has been inferred to be $S F R_{[\mathrm{OII}]}=35_{-25}^{+178} M_{\odot} \mathrm{yr}^{-1}$ (Vreeswijk et al. 2001). The large errorbars are due to the uncertainty in the estimate of the optical extinction.

To obtain an independent estimate of the $S F R$ in the host galaxy of GRB 990712, we performed $1.4 \mathrm{GHz}$ $(21 \mathrm{~cm})$ observations with the Australia Telescope Compact Array (ATCA) in March 2001. The radio continuum flux of a normal galaxy (i.e. a galaxy that is not hosting an AGN) is thought to be produced by synchrotron radiation from electrons which are accelerated by supernova remnants, and free-free emission from Hit regions (Condon 1992). The radio continuum emission should therefore be well-correlated with very recent star formation, which is strongly supported by the observed far-infrared/radio correlation. The obvious advantage of this method over the optical emission-line measurements is that the radio flux is unaffected by dust extinction, allowing an unobscured view of the star-formation nature of the GRB host.

\section{Observations}

The host galaxy of GRB 990712 was observed with the Australia Telescope Compact Array (ATCA) between 2001 March 29, 15:06 (MJD 51997.63) and March 30 03:22 (MJD 51998.14). The observations were performed in the $6 \mathrm{D}$ antenna configuration, in two bands centred at 1344 and $1432 \mathrm{MHz}$ respectively, with a total observing time on-source of $10.18 \mathrm{hr}$. Absolute flux calibration was achieved using PKS 1934-638; PKS 2101-715 was used as the phase calibrator. Data reduction was performed

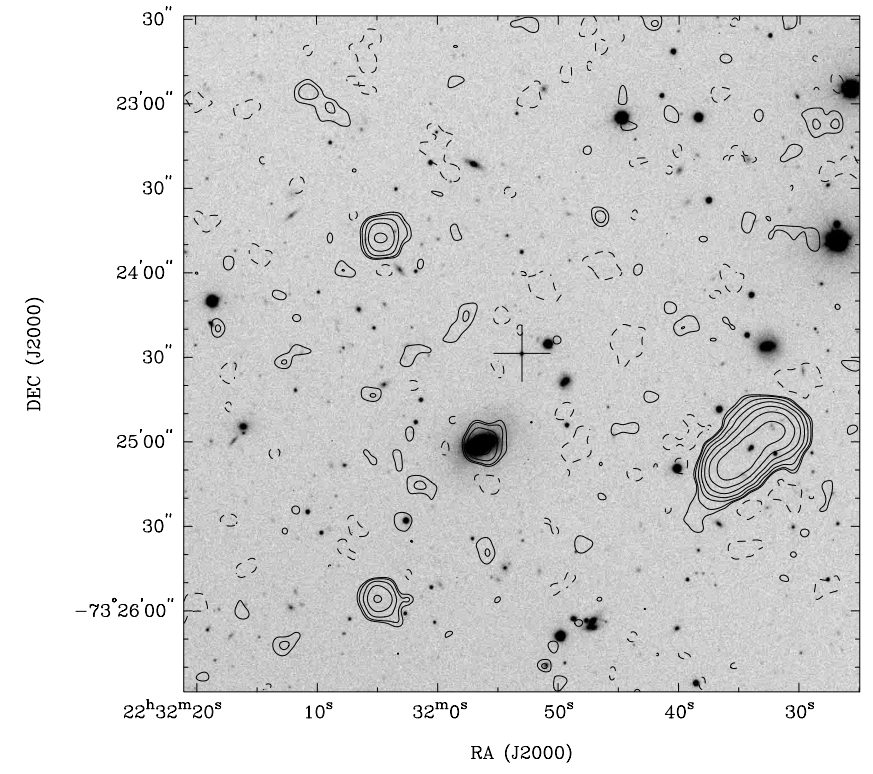

Fig. 1. The field of the host galaxy of GRB 990712 in the optical (greyscale) and radio (contours). The $R$-band image was taken with the VLT on 13 July 1999 (Sahu et al. 2000), only 0.5 days after the burst when the optical counterpart still outshone its host galaxy. Its position is indicated with a cross, The plotted radio contours are factors of -2 (dashed), 2, 3, 6 , $12,24,48$, and 96 (all solid) times the noise level of $35 \mu \mathrm{Jy}$. No $1.4 \mathrm{GHz}$ source is detected above $2 \sigma$ at the position of GRB 990712 .

using the MIRIAD software (Sault et al. 1995). Figure 1 shows a uniformly-weighted map of the region around the host galaxy with a beam size of $7.7 \times 8.8$ arcsec, superposed on an optical image (Sahu et al. 2000) taken with ESO's Very Large Telescope (VLT). No source is detected at the host galaxy location to a $2 \sigma$ limit of $70 \mu \mathrm{Jy}-$ the noise level of $35 \mu \mathrm{Jy}$ was estimated from measuring the sky around the target region. The theoretical noise limit for the observing time, bandwidth, frequency and array used is $20 \mu \mathrm{Jy} /$ beam, i.e. less than a factor of two lower than we obtain. We were unable to achieve a lower noise level using natural weighting because of sidelobes from bright, nearby sources.

\section{Results}

For a normal star-forming galaxy, the radio continuum emission is proportional to the star-formation rate (Condon 1992):

$S F R_{0.1-100 M_{\odot}}=\frac{L_{\nu, \mathrm{obs}}\left[\frac{1.4}{(1+z) \nu_{\mathrm{GHz}}}\right]^{\alpha} \times 4 \pi d_{1}^{2} \times 5.5}{5.3 \times 10^{28} \nu_{\mathrm{GHz}}^{\alpha}+5.5 \times 10^{27} \nu_{\mathrm{GHz}}^{-0.1}}$

where $L_{\nu, \mathrm{obs}}$ is the observed luminosity in $\mu \mathrm{Jy}$ at $\nu_{\mathrm{GHz}}$ and the factor $\left[\frac{1.4}{(1+z) \nu_{\mathrm{GHz}}}\right]^{\alpha}$ converts this to the emitted luminosity at $1.4 \mathrm{GHz}$ (see Haarsma et al. 2000); a is the spectral index (with $S_{\nu} \propto \nu^{\alpha}$ ), and $d_{1}$ is the luminosity distance in $\mathrm{cm}$. The first term in the denominator represents the dominant synchrotron component 
from electrons accelerated in supernova shocks; the second term comes from the thermal emission from HiI regions (for a derivation of both components, see Condon 1992). Strong evidence for this relation is supplied by the correlation between the $S F R$ determined through Eq. (1) and that obtained from the far-infrared luminosity (see e.g. Fig. 1 of Cram et al. 1998), which extends over four orders of magnitude (see also Condon 1992, and references therein). The expected lifetime of the remnant synchrotron emission is $\leq 10^{8}$ years, and thus radio continuum emission is a good tracer of very recent star formation. The original Condon relation is valid for stars with masses above $5 M_{\odot}$. Haarsma et al. (2000) extend this to the range $0.1-100 M_{\odot}$ by multiplying with a factor $Q=5.5$, which assumes a Salpeter initial mass function (IMF), i.e. $\psi(M) \propto M^{-2.35}$. This factor becomes 5.3 if an upper mass cut-off of $125 M_{\odot}$ is used, whereas it is 3.8 if the range of masses is $0.25-100 M_{\odot}$. Changing the IMF slope with \pm 0.1 (Elmegreen 1999) results in the range $Q=4-7.5$. For starburst regions, where the IMF may be weighted toward high-mass stars (Elmegreen 1999), $Q$ may be as low as unity. We take $Q=5.5$.

The resulting value for the star-formation rate that we obtain depends on the assumed spectral index and the luminosity distance, hence on the adopted cosmology. We therefore calculate the $S F R$ upper limit with the spectral index ranging from -0.35 to -1.0 , typical for normal radio galaxies, and the cosmologies $\left(h, \Omega_{\mathrm{m}}\right.$, $\left.\Omega_{\lambda}\right)=(0.65,1,0),(0.65,0.2,0)$, and $(0.65,0.3,0.7)$, where $h$ is the dimensionless Hubble constant, defined as: $h=\frac{H_{0}}{100 \mathrm{~km} \mathrm{~s}^{-1} \mathrm{Mpc}^{-1}}$. The redshift of the GRB and its host galaxy is $0.4331 \pm 0.0004$ (Vreeswijk et al. 2001). The resulting luminosity distances (see Hogg 1999) for these cosmologies are respectively: $6.7 \times 10^{27} \mathrm{~cm}, 7.3 \times 10^{27} \mathrm{~cm}$ and $7.9 \times 10^{27} \mathrm{~cm}$. Inserting our $2 \sigma$ upper limit of $70 \mu \mathrm{Jy}$, and the range in spectral indices and luminosity distances, we obtain the following range for the upper limit on the $S F R$ in the host of GRB 990712: $S F R_{\max }=47-100 M_{\odot} \mathrm{yr}^{-1}$.

\section{Discussion}

Table 1 shows the GRB host galaxies for which an [OII] emission-line flux has been reported in the literature. The $\mathrm{H} \alpha$ line emission is a more reliable optical estimate of the $S F R$ than [OII], but this line is usually shifted into the near-infrared passbands, making it observationally difficult to obtain the $\mathrm{H} \alpha$ line flux. The listed [OII] flux, which is corrected for the Galactic foreground extinction, is taken from the references indicated in the caption. We convert this flux, using $\left(h, \Omega_{\mathrm{m}}, \Omega_{\lambda}\right)=(0.65,0.3,0.7)$ and the calibration from Kennicutt (1998), to obtain an estimate of the attenuated $S F R$ (Col. 4), i.e. not corrected for dust extinction. The error in this conversion is roughly $30 \%$. With $\left(h, \Omega_{\mathrm{m}}, \Omega_{\lambda}\right)=(0.65,0.2,0)$, these $S F R$ estimates need to be scaled down by about $20 \%$.

The [OII] emission line method shows that GRB host galaxies differ widely in their star-formation nature, from 0.3 to $24 M_{\odot} \mathrm{yr}^{-1}$, i.e. a factor of 80 . However, extinction is an important factor in optical $S F R$ indicators, which could boost these estimates up to much higher values. A good example is the host galaxy featured in this paper: GRB 990712. Vreeswijk et al. (2001) estimate the SFR of the host of GRB 990712 to be $35_{-25}^{+178} M_{\odot} \mathrm{yr}^{-1}$ from the [OII] emission line, $64_{-54}^{+770} M_{\odot} \mathrm{yr}^{-1}$ from $\mathrm{H} \beta$ and $\sim 400 M_{\odot} \mathrm{yr}^{-1}$ from the $2800 \AA$ flux. These values reflect the large uncertainty in the dust extinction estimate for this host, obtained from the observed and expected ratio of $\mathrm{H} \beta$ and $\mathrm{H} \gamma$. The radio data presented in this paper provide a clear upper limit to these $S F R$ s of $100 M_{\odot} \mathrm{yr}^{-1}$, indicating that the extinction at $2800 \AA$ has been overestimated. In Table 1 we also list the extinction-corrected SFRs, using host-galaxy extinction measurements from the literature (Sokolov et al. 2001; Vreeswijk et al. 2001). Note that the value for the host of GRB 990712 differs from that reported by Vreeswijk et al. (2001) due to the different cosmologies used.

Recently, very high $S F R$ s have been inferred for two other GRB host galaxies, not from the optical but through two methods that are not affected by the dust along the line of sight. From millimeter and submillimeter wavelength observations, Frail et al. (2001) infer a rate of $\sim 600 M_{\odot} \mathrm{yr}^{-1}$ for the host of GRB 010222. The host of this burst is faint: $V=26.0 \pm 0.1$ (Fruchter et al. 2001), making it difficult to obtain an [OII] flux measurement. Berger et al. (2001) measure a $1.4 \mathrm{GHz}$ flux of $68.0 \pm 6.6 \mu \mathrm{Jy}$ for the host of GRB $980703(z=0.966)$, and infer a star-formation rate of $\sim 500 M_{\odot} \mathrm{yr}^{-1}$, with the same method and assumptions as we employ in this paper. The latter authors use the empirical relation between the far infra-red (FIR) and radio emission (van der Kruit 1971; Helou et al. 1985), to estimate the FIR luminosity of the host of GRB 980703 to be that of an ultraluminous infra-red galaxy (ULIG or ULIRG, for which $\left.L_{\mathrm{IR}}>10^{12} L_{\odot}\right)$. Applying the same relation to our $1.4 \mathrm{GHz}$ flux of GRB 990712 (using $d_{\mathrm{l}}=7.9 \times 10^{27} \mathrm{~cm}$ ), we find $L_{\mathrm{IR}}<1.1 \times 10^{11} L_{\odot}$, more than a factor of 20 less luminous in the IR than the host of GRB 980703. This indicates that the host of GRB 990712 does not belong to the class of ULIRGs, although direct IR observations would be needed to definitely rule out the possibility.

Our observations of the host of GRB 990712 present the deepest radio-determined $S F R$ limit on a GRB host galaxy yet, and show that also when observed at unobscured radio wavelengths, not every host is a vigorous starburst galaxy. How do the [OII] and radio $S F R$ estimates compare in general? The optical $S F R$ estimators, after correcting for internal extinction, tend to underestimate the total $S F R$, when compared to the FIR and radio methods (e.g. Cram et al. 1998). The host of GRB 980703 is a striking example of this: the extinction-corrected $S F R$ is about a factor of 15 lower than the rate estimated from the radio continuum flux (see Table 1). We therefore use the prescription of Hopkins et al. (2001, Eq. (5)) to convert the attenuated $S F R$ estimates of Col. 4 in Table 1 to total $S F R$ s (Col. 7). This conversion is based on an empirical 
Table 1. Star-formation rate estimates from the [OII] nebular emission-line and $1.4 \mathrm{GHz}$ flux; for both a Salpeter IMF over the mass range $0.1-100 M_{\odot}$ is assumed. The [OII] fluxes (corrected for the Galactic foreground extinction), are taken from the following references: 1) Bloom et al. (2001a); 2) Bloom et al. (1998); 3) Djorgovski et al. (2001); 4) Djorgovski et al. (1999); 5) Djorgovski et al. (1998); 6) Vreeswijk et al. (2001); 7) Castro-Tirado et al. (2001); 8) Price et al. (2001). We use the currently popular cosmology $\left(h, \Omega_{\mathrm{m}}, \Omega_{\lambda}\right)=(0.65,0.3,0.7)$ to obtain the luminosity distance (see also Bloom et al. 2001b), in order to convert the line flux into luminosity. We apply the conversion from Kennicutt (1998) to this [OII] luminosity to obtain the attenuated $S F R \mathrm{~s}$, listed in Col. 4. The error in this conversion is roughly 30\% (Kennicutt 1998). We correct these $S F R$ s in two different ways. First, we use the host extinction measurements from Sokolov et al. (2001) and Vreeswijk et al. (2001), and scale the attenuated $S F R$ to the extinction-corrected $S F R$ of Col. 5. Second, the attenuated $S F R$ estimates are also corrected in a more general way, using the prescription of Hopkins et al. (2001) for the relation between the $S F R$ obtained from optical emission lines, and the $S F R$ obtained from the $1.4 \mathrm{GHz}$ flux for a sample of radio galaxies. These estimates are shown in Col. 7 . The scatter in this conversion from attenuated to corrected $S F R$ (see Fig. 3 of Hopkins et al. 2001) is approximately a factor of 2 to 3. The radio-determined $S F R$ s are listed in Col. 6.

\begin{tabular}{|c|c|c|c|c|c|c|}
\hline host of GRB & $\begin{array}{c}{[\mathrm{OII}] \text { flux }} \\
\left(10^{-16} \mathrm{erg} \mathrm{s}^{-1} \mathrm{~cm}^{-2}\right)\end{array}$ & reference & $\begin{array}{c}S F R_{[\mathrm{OII}]-\text { att. }} \\
\quad M_{\odot} \mathrm{yr}^{-1}\end{array}$ & $\begin{array}{l}S F R_{[\mathrm{OII}]-\text { corr. }} \\
M_{\odot} \mathrm{yr}^{-1}\end{array}$ & $\begin{array}{c}S F R_{1.4 \mathrm{GHz}}^{\mathrm{d}} \\
M_{\odot} \mathrm{yr}^{-1}\end{array}$ & $\begin{array}{c}S F R_{[\mathrm{OII}]-\text { Hopkins }} \\
M_{\odot \mathrm{yr}^{-1}}\end{array}$ \\
\hline 970228 & $0.22 \pm 0.01$ & 1 & 0.76 & - & $<380^{\mathrm{e}}$ & 5.5 \\
\hline 970508 & $0.30 \pm 0.02$ & 2 & 1.6 & $5.8^{\mathrm{b}}$ & $<430^{\mathrm{f}}$ & 17 \\
\hline 970828 & $0.045 \pm 0.01^{\mathrm{a}}$ & 3 & 0.3 & - & $<360^{\mathrm{g}}$ & 1.2 \\
\hline 980613 & 0.44 & 4 & 4.7 & $21^{\mathrm{b}}$ & - & 82 \\
\hline 980703 & 3.04 & 5 & 24 & $34^{\mathrm{b}}$ & $\approx 500$ & 784 \\
\hline 990712 & $3.32 \pm 0.14$ & 6 & 3.7 & $44^{\mathrm{c}}$ & $<100$ & 58 \\
\hline 991208 & $1.79 \pm 0.22$ & 7 & 6.4 & $23^{\mathrm{b}}$ & - & 127 \\
\hline 000911 & $0.23 \pm 0.03$ & 8 & 2.2 & - & - & 28 \\
\hline
\end{tabular}

a Assuming galaxy B of Djorgovski et al. (2001) is the host. Inclusion of galaxy A would increase the [OII] flux and the attenuated $S F R$ with a factor of about 5, resulting in a corrected $S F R$ of 17 (Col. 7 ).

b Adopting the host extinction as determined by Sokolov et al. (2001).

c Adopting the host extinction as determined by Vreeswijk et al. (2001), who used $\left(h, \Omega_{\mathrm{m}}, \Omega_{\lambda}\right)=(0.70,0.3,0)$, which results in a lower $S F R$ of $35 M_{\odot} \mathrm{yr}^{-1}$.

d All upper limits are $2 \sigma$, and we assume $\alpha=-1$ and $\left(h, \Omega_{\mathrm{m}}, \Omega_{\lambda}\right)=(0.65,0.3,0.7)$.

e Taking the estimated $2 \sigma$ upper limit of $71 \mu \mathrm{Jy}$ from Frail et al. (1998) and $z=0.695$.

$\mathrm{f}$ Taking the estimated $2 \sigma$ upper limit of $45 \mu \mathrm{Jy}$ from Frail et al. (2000) and $z=0.835$.

$\mathrm{g}$ Taking the estimated $2 \sigma$ upper limit of $26 \mu \mathrm{Jy}$ from Djorgovski et al. (2001) and $z=0.958$.

correlation between obscuration and far-infrared luminosity (Wang \& Heckman 1996), from which Hopkins et al. (2001) deduce a relation between obscuration and $S F R$. These numbers can now be compared with $S F R$ methods that do not suffer from dust extinction, such as the 1.4 GHz continuum flux method. For the two host galaxies for which these numbers are available, the values are consistent within the (large) errors.

A large sample of GRBs with redshift determinations (through e.g. rapid spectroscopy of the afterglow) and 1.4 GHz or submillimeter observations of their hosts, can provide an important step toward calibration of the possible relation between GRB number counts and the total star-formation density as a function of look-back time. Different classes of GRBs, if produced by different progenitors, could be used to verify this calibration.

Acknowledgements. PMV is supported by the NWO Spinoza grant $08-0$ to E. P. J. van den Heuvel. LK is supported by a fellowship of the Royal Academy of Arts \& Sciences in The Netherlands. The Australia Telescope Compact Array is part of the Australia Telescope which is funded by the Commonwealth of Australia for operation as a National Facility managed by CSIRO. PMV kindly thanks David Hogg for providing his code (see Hogg 1999) to verify the obtained luminosity distances. PMV also thanks E. P. J. van den Heuvel for valuable comments.

\section{References}

Berger, E., Kulkarni, S. R., \& Frail, D. A. 2001, ApJ, 560, 652 Bloom, J. S., Djorgovski, S. G., \& Kulkarni, S. R. 2001a, ApJ, 554,678

Bloom, J. S., Djorgovski, S. G., Kulkarni, S. R., \& Frail, D. A. 1998, ApJ, 507, L25

Bloom, J. S., Frail, D. A., \& Sari, R. 2001b, AJ, 121, 2879

Bloom, J. S., Kulkarni, S. R., \& Djorgovski, S. G. 2001c, ApJ, submitted [astro-ph/0010176]

Bloom, J. S., Kulkarni, S. R., Djorgovski, S. G., et al. 1999a, Nature, 401, 453

Bloom, J. S., Sigurdsson, S., \& Pols, O. R. 1999b, MNRAS, 305,763

Castro-Tirado, A. J., Sokolov, V. V., Gorosabel, J., et al. 2001, A\&A, 370, 398

Condon, J. J. 1992, ARA\&A, 30, 575

Cram, L., Hopkins, A., Mobasher, B., \& Rowan-Robinson, M. 1998, ApJ, 507, 155

Djorgovski, S. G., Frail, D. A., Kulkarni, S. R., et al. 2001, ApJ, submitted [astro-ph/0107539] 
Djorgovski, S. G., Kulkarni, S. R., Bloom, J. S., et al. 1999, GRB Circ. Net., 189

Djorgovski, S. G., Kulkarni, S. R., Bloom, J. S., et al. 1998, ApJ, 508, L17

Eichler, D., Livio, M., Piran, T., \& Schramm, D. N. 1989, Nature, 340, 126

Ellis, R. S. 1997, ARA\&A, 35, 389

Elmegreen, B. G. 1999, ApJ, 515, 323

Frail, D. A., Bertoldi, G. H., Moriarty-Schieven, G. H., et al. 2001, ApJ, submitted [astro-ph/0108436]

Frail, D. A., Kulkarni, S. R., Shepherd, D. S., \& Waxman, E. 1998, ApJ, 502, L119

Frail, D. A., Waxman, E., \& Kulkarni, S. R. 2000, ApJ, 537, 191

Fruchter, A., Burud, I., Rhoads, J., \& Levan, A. 2001, GRB Circ. Net., 1087

Fryer, C. L., Woosley, S. E., \& Hartmann, D. H. 1999, ApJ, 526,152

Galama, T. J., Tanvir, N., Vreeswijk, P. M., et al. 2000, ApJ, 536,185

Galama, T. J., Vreeswijk, P. M., van Paradijs, J., et al. 1998, Nature, 395, 670

Haarsma, D. B., Partridge, R. B., Windhorst, R. A., \& Richards, E. A. 2000, ApJ, 544, 641

Helou, G., Soifer, B. T., \& Rowan-Robinson, M. 1985, ApJ, 298, L7
Hogg, D. W. 1999, [astro-ph/9905116]

Hopkins, A. M., Connolly, A. J., Haarsma, D. B., \& Cram, L. E. 2001, AJ, 122, 288

Kennicutt, R. C. 1998, ARA\&A, 36, 189

MacFadyen, A. I. \& Woosley, S. E. 1999, ApJ, 524, 262

MacFadyen, A. I., Woosley, S. E., \& Heger, A. 2001, ApJ, 550, 410

Narayan, R., Paczyński, B., \& Piran, T. 1992, ApJ, 395, L83

Paczyński, B. 1998, ApJ, 494, L45

Price, P. A., Berger, E., Kulkarni, S. R., et al. 2001, ApJ, submitted [astro-ph/0110315]

Reichart, D. E. 1999, ApJ, 521, L111

Sahu, K. C., Vreeswijk, P., Bakos, G., et al. 2000, ApJ, 540, 74

Sanders, D. B., \& Mirabel, I. F. 1996, ARA\&A, 34, 749

Sault, R. J., Teuben, P. J., \& Wright, M. C. H. 1995, in Astron. Data Anal. Soft. Syst. IV, vol. 4, ASP Conf. Ser., 77, 433

Sokolov, V. V., Fatkhullin, T. A., Castro-Tirado, A. J., et al. 2001, A\&A, 372, 438

Stratta, G., Piro, L., Gandolfi, G., et al. 2000, in Gamma-ray Bursts, 5th Huntsville Symp., AIP Conf. Proc., 526, 375

van der Kruit, P. C. 1971, A\&A, 15, 110

Vreeswijk, P. M., Fruchter, A., Kaper, L., et al. 2001, ApJ, 546, 672

Wang, B., \& Heckman, T. M. 1996, ApJ, 457, 645

Wijers, R. A. M. J., \& Galama, T. J. 1999, ApJ, 523, 177

Woosley, S. E. 1993, ApJ, 405, 273 